\title{
An eigenfunction expansion approach for the derivation of asymptotic expansions in financial valuation problems
}

\author{
Manabu Miyamoto ${ }^{1,2 *}$ and Keiichi Tanaka ${ }^{2}$ \\ ${ }^{1}$ Mizuho Securities Co. Ltd., Otemachi First Square 1-5-1, Otemachi, Chiyoda-ku, Tokyo 100- \\ 0004, Japan \\ 2 Tokyo Metropolitan University, 1-1 Minami-Ohsawa, Hachioji, Tokyo 192-0397, Japan \\ *Corresponding author: manabu.miyamoto@mizuho-sc.com
}

Received November 03, 2021, Accepted January 13, 2022

\begin{abstract}
This study examines whether an alternative calculation approach exists for the asymptotic expansion of the probability density function of risky asset prices. We focus on the partial differential equation by using an eigenfunction expansion related to Hermite polynomials. We show that the expansion coefficients can be obtained by solving ordinary differential equations recursively and which order of Hermite polynomials appear in asymptotic expansion explicitly.
\end{abstract}

Keywords asymptotic expansion, eigenfunction expansion, financial valuation problem

Research Activity Group Mathematical Finance

\section{Introduction}

Based on derived analytic formulas, asymptotic expansion techniques benefit financial institutions not only in terms of the real-time pricing of exotic products but also in the fast calibrations of model parameters $[1,2]$. Despite being powerful, this method sometimes requires a large number of calculations including conditional expectations of products of the asymptotic expansion coefficients of underlying asset price. Some recent developments [2] reveal that conditional expectations satisfy a finite system of ordinary differential equations (ODEs) that can reduce practitioners' calculation efforts.

Some complexity of the calculation may be caused by starting from an asymptotic expansion of the asset price. Therefore in this letter we focus on the partial differential equation (PDE) determining the probability density function $(\mathrm{PDF})$, without any resort to an expansion of the asset price itself. By restricting ourselves to a BlackScholes economy with one risky asset, we then show that by applying the eigenfunction expansion with Hermite polynomials, the asymptotic expansion coefficients are recursively determined by the ODEs and the resulting asymptotic expansion of PDF matches to that derived by $[1,2]$ up to second order approximation. Furthermore, through the orthogonality of Hermite polynomials, we reveal which order of Hermite polynomials appear in the asymptotic expansion explicitly. An approach based on the PDE has already been proposed in the literature (see [3] and the references therein). However, to the best of our knowledge, there is no investigation into the relationship between the PDE and the eigenfunction expansion by Hermite polynomials.

\section{Model and settings}

We consider a Black-Scholes economy with a finite time horizon, $\mathbb{T}=[0, T]$ on a filtered probability space,
$\left(\Omega, \mathcal{F}, Q,\left\{\mathcal{F}_{t}\right\}_{t \in \mathbb{T}}\right)$ where $W=\left\{W_{t}\right\}_{t \in \mathbb{T}}$ is equipped as the standard Brownian motion under risk neutral measure $Q$ and $\mathcal{F}_{t}$ is given by $\sigma\left\{W_{s} \mid 0 \leq s \leq t\right\}$, by following the settings in [1]. Price $S_{t}^{(\epsilon)}$ of a risky asset at time $t$ is described by the one-dimensional stochastic differential equation (SDE)

$$
d S_{t}^{(\epsilon)}=r(t) S_{t}^{(\epsilon)} d t+\epsilon \sigma\left(t, S_{t}^{(\epsilon)}\right) d W_{t}, \quad S_{0}^{(\epsilon)}:=S_{0},
$$

where $r$ is a bounded and deterministic function of time $t$ and represents the process of the instantaneous interest rate and $\epsilon(>0)$ is the expansion parameter. It is useful to convert $S_{t}^{(\epsilon)}$ to a normalized price process $\left\{X_{t}^{(\epsilon)}\right\}_{t \in \mathbb{T}}$ defined by $X_{t}^{(\epsilon)}:=\left(S_{t}^{(\epsilon)}-F(0, t)\right) / \epsilon$ with $X_{0}^{(\epsilon)}=0$, where $F(0, t):=E\left[S_{t}^{(\epsilon)}\right]=S_{0} \exp \left(\int_{0}^{t} r(u) d u\right)$ is the forward value of $S_{t}^{(\epsilon)}$ and symbol $E$ denotes the expectation under $Q$. Then, we see that

$$
d X_{t}^{(\epsilon)}=r(t) X_{t}^{(\epsilon)} d t+\sigma\left(t, F(0, t)+\epsilon X_{t}^{(\epsilon)}\right) d W_{t} .
$$

A process $\left\{X_{t}^{(0)}\right\}_{t \in \mathbb{T}}$ is defined by the solution of (2) with $\epsilon=0$ and $X_{0}^{(0)}=0$. Then it is clear that $X_{t}^{(0)}$ is normally distributed $N(0, \Sigma(t))$ where $\Sigma(t):=$ $e^{2 \int_{0}^{t} r(s) d s} \int_{0}^{t} e^{-2 \int_{0}^{u} r(s) d s} \sigma^{2}(u, F(0, u)) d u$.

\section{Review of derivation based on SDE}

We briefly review the derivation of the asymptotic expansion in the literature by following $[1,2]$ on the same setting as above. Note that main concerns of $[1,2]$ are asymptotic expansions of stochastic processes and an expansion of PDF is obtained as a result. $X_{t}^{(\epsilon)}$ is expanded by $\epsilon$ as

$$
X_{t}^{(\epsilon)}=X_{t}^{(0)}+\sum_{k=2}^{N+1} \epsilon^{k-1} A_{k t}+o\left(\epsilon^{N}\right)
$$


as $\epsilon \downarrow 0$ in $\mathbf{D}^{\infty}$ where $\mathbf{D}^{\infty}$ is the set of smooth Wiener functionals (in the sense of Malliavin). Each of $\left\{A_{k t}\right\}_{t \in \mathbb{T}}$ obeys an appropriate SDE derived from (2). Then it is found that $A_{k t}$ is recursively determined as a multiple integral of lower order solutions $\left\{A_{l t}\right\}_{l=1}^{k-1}$. Using this expansion (3), it follows that the asymptotic expansion of $\operatorname{PDF} p^{(\epsilon)}(t, y):=\partial_{y} E\left[1_{\left\{X_{t}^{(\epsilon)} \leq y\right\}}\right]$, where $\partial_{y}^{n}=\partial^{n} / \partial y^{n}$, up to second order is obtained as

$$
\begin{gathered}
p^{(\epsilon)}(t, y)=p^{(0)}(t, y)-\epsilon \partial_{y}\left\{E\left[A_{2 t} \mid X_{t}^{(0)}=y\right] p^{(0)}(t, y)\right\} \\
+\epsilon^{2} \frac{1}{2 !} \partial_{y}^{2}\left\{E\left[\left(A_{2 t}\right)^{2} \mid X_{t}^{(0)}=y\right] p^{(0)}(t, y)\right\} \\
-\epsilon^{2} \partial_{y}\left\{E\left[A_{3 t} \mid X_{t}^{(0)}=y\right] p^{(0)}(t, y)\right\} \\
+o\left(\epsilon^{2}\right) .
\end{gathered}
$$

Conditional expectations on the right-hand side (RHS) are calculated either by making use of the representation of $A_{k t}$ as a multiple integral as shown in [1], or by the following fact on a conditional expectation of a random variable $X \in L^{2}(\Omega, Q)$ with respective to a normal random variable $Z \sim N(0, \Sigma)$ as shown in [2]

$$
\begin{aligned}
& E[X \mid Z=x]=\sum_{n=0}^{\infty} \frac{a_{n}}{\Sigma^{n}} H_{n}(x ; \Sigma), \\
& a_{n}:=\left.\frac{1}{i^{n} n !} \partial_{\xi}^{n}\left\{e^{\frac{\xi^{2}}{2} \Sigma} E\left[e^{i \xi Z} X\right]\right\}\right|_{\xi=0},
\end{aligned}
$$

where $H_{n}(x ; \Sigma):=(-\Sigma)^{n} e^{x^{2} / 2 \Sigma} \frac{d^{n}}{d x^{n}} e^{-x^{2} / 2 \Sigma}$ for $n \geq 0$. In the latter case, by substituting (5) with $X=\left(A_{k t}\right)^{j}$ and $Z=X_{t}^{(0)}$ one can express the asymptotic expansion (4) as a sum of Hermite polynomials (See Theorem 3.2 in [2]) though the coefficients still remain to be calculated. Then, the authors show that these expectations in the coefficients satisfy a finite system of ODEs. The recursive structure of $A_{k t}$ is inherited by the solution of the ODEs. In either case, further investigations are required in order to better understand the expansion (4) in a general way and apply it to an efficient pricing of derivative securities, that is our motivation.

\section{A new derivation based on PDE}

We alternatively focus on the $\operatorname{PDE}$ of $p^{(\epsilon)}(t, y)$. Corresponding to SDE (2), the Kolmogorov forward equation becomes

$$
\left(\partial_{t} p^{(\epsilon)}\right)(t, y)=\left(\mathcal{L}^{(\epsilon)} p^{(\epsilon)}\right)(t, y), \quad p^{(\epsilon)}(0, y)=\delta(y),
$$

where $\mathcal{L}^{(\epsilon)}$ is the formal adjoint of the generator of $X_{t}^{(\epsilon)}$ defined by

$$
\left(\mathcal{L}^{(\epsilon)} f\right)(y):=(1 / 2) \partial_{y}^{2}(\alpha(t, \epsilon y) f(y))-\partial_{y}(r(t) y f(y))
$$

and $\alpha(t, y):=\sigma^{2}(t, F(0, t)+y)$. By considering $X_{t}^{(\epsilon)} \rightarrow$ $X_{t}^{(0)}$ when $\epsilon \downarrow 0$ in $\mathbf{D}^{\infty}$, we want to find the solution satisfying

$$
\lim _{\epsilon \downarrow 0} p^{(\epsilon)}(t, y)=p^{(0)}(t, y), \quad \forall t>0, \forall y \in \mathbb{R} .
$$

In response to the fact $X_{t}^{(0)} \sim N(0, \Sigma(t))$, the PDE for $\epsilon=0$ has the solution identical to the PDF of the normal distribution

$$
p^{(0)}(t, y)=(2 \pi \Sigma(t))^{-1 / 2} \exp \left(-y^{2} /(2 \Sigma(t))\right) .
$$

In order to solve PDE (6), let us consider a factorization with $p^{(0)}(t, y)$ as

$$
p^{(\epsilon)}(t, y)=g^{(\epsilon)}(t, y) p^{(0)}(t, y)
$$

where $g^{(\epsilon)}(t, y)$ is assumed to be formally expanded with $g_{n}(t, y)=\left.\partial_{\epsilon}^{n} g^{(\epsilon)}(t, y)\right|_{\epsilon \downarrow 0}$ by the power series of $\epsilon$ as

$$
g^{(\epsilon)}(t, y)=\sum_{n=0}^{\infty} \frac{\epsilon^{n}}{n !} g_{n}(t, y)
$$

Therefore, we concentrate on $g^{(\epsilon)}$ and $g_{n}$ in what follows. First, let us check the conditions under which $\epsilon \downarrow 0$ or $t \downarrow 0$. It is apparent that the behavior of $g^{(\epsilon)}(t, y)$ when $\epsilon \downarrow 0$ or $t \downarrow 0$ is related to the ones of $p^{(\epsilon)}(t, y)$ and $p^{(0)}(t, y)$. By inserting (10) and (11) into (8), we observe that

$$
\lim _{\epsilon \downarrow 0} g^{(\epsilon)}(t, y)=g_{0}(t, y)=1, \quad \forall t>0, \forall y \in \mathbb{R} .
$$

For the behavior when $t \downarrow 0$, the initial condition of (6) means that $h(0)=\lim _{t \downarrow 0} \int_{-\infty}^{\infty} h(y) p^{(\epsilon)}(t, y) d y, \forall \epsilon>0$, for some class of the payoff function $h$. By inserting (10) and (11) into this condition and making use of $(12)\left(g_{0} \equiv\right.$ 1 ), we have

$$
\lim _{t \downarrow 0} \int_{-\infty}^{\infty} h(y) g_{n}(t, y) p^{(0)}(t, y) d y=0
$$

for $n=1,2, \ldots$.

Next, we obtain the PDE that essentially derives $g_{n}$. For this purpose, it is convenient to change variables $(t, y)$ to new ones, $(t, \eta)$ with $\eta:=y / \sqrt{\Sigma(t)}$ by reflecting the functional form of (9). $\eta$ could be interpreted as the normalized position at time $t$ by the standard deviation. Then, the differentiation by $y$ becomes $\partial_{y}=(\Sigma(t))^{-1 / 2} \partial_{\eta}$. Corresponding to this change, we rewrite the density functions as $\psi^{(\epsilon)}(t, \eta):=p^{(\epsilon)}(t, \sqrt{\Sigma(t)} \eta) \sqrt{\Sigma(t)}$. For other relevant functions, we set $\psi^{(0)}(t, \eta):=p^{(0)}(t, \sqrt{\Sigma(t)} \eta) \sqrt{\Sigma(t)}=$ $(2 \pi)^{-1 / 2} \exp \left(-\eta^{2} / 2\right)=\psi^{(0)}(\eta), \quad \varphi^{(\epsilon)}(t, \eta) \quad:=$ $g^{(\epsilon)}(t, \sqrt{\Sigma(t)} \eta)$, and $\varphi_{n}(t, \eta):=g_{n}(t, \sqrt{\Sigma(t)} \eta)$, respectively. The diffusion coefficient $\alpha(t, y)$ is also expanded as $\alpha(t, \epsilon y)=\sum_{n=0}^{\infty}(\epsilon y)^{n} / n !\left(\partial_{y}^{n} \alpha\right)(t, 0)$. Вy substituting this expression into (7), we formally expand $\mathcal{L}^{(\epsilon)}$ as

$$
\mathcal{L}^{(\epsilon)}=\sum_{n=0}^{\infty} \frac{\epsilon^{n}}{n !} \mathcal{L}_{n}
$$

where $\mathcal{L}_{0}=\mathcal{L}^{(0)}$ and $\left(\mathcal{L}_{n} f\right)(y)=$ $(1 / 2)\left(\partial_{y}^{n} \alpha\right)(t, 0) \partial_{y}^{2}\left(y^{n} f(y)\right)$ for $n=1,2, \ldots$ In terms of $\eta$, these differential operators become

$$
\left(\mathcal{L}_{n} \varphi\right)(\eta)=\left\{\begin{array}{cc}
\frac{\alpha(t, 0)}{2 \Sigma(t)} \partial_{\eta}^{2} \varphi(\eta)-r(t) \partial_{\eta}(\eta \varphi(\eta)) & \text { if } n=0, \\
\frac{\left(\partial_{y}^{n} \alpha\right)(t, 0)}{2 \Sigma(t)}(\Sigma(t))^{n / 2} \partial_{\eta}^{2}\left(\eta^{n} \varphi(\eta)\right) & \text { if } n \geq 1,
\end{array}\right.
$$

for a suitable function $\varphi$ of $\eta$.

Furthermore, let us introduce operators $\mathcal{B}_{\eta}^{ \pm}$defined as

$$
\mathcal{B}_{\eta}^{+} \varphi(\eta):=-\partial_{\eta} \varphi(\eta)+\eta \varphi(\eta), \quad \mathcal{B}_{\eta}^{-} \varphi(\eta):=\partial_{\eta} \varphi(\eta) .
$$


Then the multiplication by $\eta$ is expressed as the sum

$$
\eta \varphi(\eta)=\left(\mathcal{B}_{\eta}^{+}+\mathcal{B}_{\eta}^{-}\right) \varphi(\eta) .
$$

Thus, one can always change the expression of pair $\left(\eta, \partial_{\eta}\right)$ to $\left(\mathcal{B}_{\eta}^{+}, \mathcal{B}_{\eta}^{-}\right)$and vice versa. $\mathcal{B}_{\eta}^{ \pm}$satisfies the commutation relation

$$
\left(\mathcal{B}_{\eta}^{-} \mathcal{B}_{\eta}^{+}-\mathcal{B}_{\eta}^{+} \mathcal{B}_{\eta}^{-}\right) \varphi(\eta)=\varphi(\eta) .
$$

Especially $\mathcal{B}_{\eta}^{ \pm}$have close relationships with the Hermite polynomials $H_{n}(\eta)=H_{n}(\eta ; 1)$ such as the properties of

$$
\mathcal{B}_{\eta}^{+} H_{n}(\eta)=H_{n+1}(\eta), \quad \mathcal{B}_{\eta}^{-} H_{n}(\eta)=n H_{n-1}(\eta),
$$

and the eigenvalue equation

$$
\mathcal{B}_{\eta}^{+} \mathcal{B}_{\eta}^{-} H_{n}(\eta)=n H_{n}(\eta) \quad(n=0,1, \ldots) .
$$

(20) implies that the eigenfunction of $\mathcal{B}_{\eta}^{+} \mathcal{B}_{\eta}^{-}$corresponding to eigenvalue $n$ is $H_{n}$. Operators $\mathcal{B}_{\eta}^{ \pm}$are inspired from the creation and annihilation operators used in quantum mechanics (e.g., [4] and references therein).

Under the preparation mentioned above, we begin by expanding the left-hand side (LHS) of (6) by $\epsilon$ with the change of variables to $(t, \eta)$. Substituting (10) and (11) into the LHS of (6), we have

$$
\begin{aligned}
& \left(\partial_{t} p^{(\epsilon)}\right)(t, y) \\
& =\sum_{n=0}^{\infty} \frac{\epsilon^{n}}{n !}\left[\left(\partial_{t} \varphi_{n}\right)(t, \eta)-\frac{1}{2} \frac{d \ln \Sigma(t)}{d t} \mathcal{B}_{\eta}^{+} \mathcal{B}_{\eta}^{-} \varphi_{n}(t, \eta)\right. \\
& \left.\quad-\frac{1}{2} \frac{d \ln \Sigma(t)}{d t}\left(\mathcal{B}_{\eta}^{-}\right)^{2} \varphi_{n}(t, \eta)\right] \frac{\psi^{(0)}(\eta)}{\sqrt{\Sigma(t)}} \\
& \quad+\left(\sum_{n=0}^{\infty} \frac{\epsilon^{n}}{n !} \varphi_{n}(t, \eta)\right)\left(\partial_{t} p^{(0)}\right)(t, \sqrt{\Sigma(t)} \eta),
\end{aligned}
$$

where we have used $\partial_{t} \eta=-(1 / 2)(d \ln \Sigma(t) / d t) \eta$ and facts (16), (18), and (17).

Our next task is the expansion of the RHS of (6) by $\epsilon$ with the change of variables to $(t, \eta)$. On substituting (10), (11), and (14) into the RHS of (6), we obtain

$$
\left(\mathcal{L}^{(\epsilon)} p^{(\epsilon)}\right)(t, y)=\sum_{n=0}^{\infty} \frac{\epsilon^{n}}{n !} \sum_{m=0}^{n}\left(\begin{array}{c}
n \\
m
\end{array}\right)\left(\mathcal{L}_{n-m} g_{m} p^{(0)}\right)(t, y) .
$$

Each term $\mathcal{L}_{n-m} g_{m} p^{(0)}$ in the above summation can be essentially replaced with terms $\left\{\left(\mathcal{B}_{\eta}^{+}\right)^{k}\left(\mathcal{B}_{\eta}^{-}\right)^{l} \varphi_{m}\right\} \psi^{(0)}$. Note, however, that (15) implies distinct forms of operator $\mathcal{L}_{n-m}$ depending on whether $n-m$ is equal to zero or not. When $m<n$, we obtain

$$
\begin{aligned}
&\left(\mathcal{L}_{n-m} g_{m} p^{(0)}\right)(t, y) \\
&= \frac{\left(\partial_{y}^{n-m} \alpha\right)(t, 0)}{2 \Sigma(t)}(\Sigma(t))^{(n-m) / 2} \\
& \quad \times\left[\left(\mathcal{B}_{\eta}^{+}\right)^{2}\left(\mathcal{B}_{\eta}^{+}+\mathcal{B}_{\eta}^{-}\right)^{n-m} \varphi_{m}(t, \eta)\right] \frac{\psi^{(0)}(\eta)}{\sqrt{\Sigma(t)}},
\end{aligned}
$$

where $(16)$ and the facts $\partial_{\eta} \psi^{(0)}(\eta)=-\eta \psi^{(0)}(\eta)$ and $\partial_{\eta}^{2} \psi^{(0)}(\eta)=\left(\eta^{2}-1\right) \psi^{(0)}(\eta)$ are used. On the other hand, when $m=n$, we observe that

$$
\left(\mathcal{L}_{0} g_{n} p^{(0)}\right)(t, y)-g_{n}(t, y)\left(\mathcal{L}_{0} p^{(0)}\right)(t, y)
$$

$$
\begin{aligned}
= & {\left[-\left(\frac{d \ln \Sigma(t)}{d t}-r(t)\right) \mathcal{B}_{\eta}^{+} \mathcal{B}_{\eta}^{-} \varphi_{n}(t, \eta)\right.} \\
& \left.-\frac{1}{2} \frac{d \ln \Sigma(t)}{d t} \times\left(\mathcal{B}_{\eta}^{-}\right)^{2} \varphi_{n}(t, \eta)\right] \frac{\psi^{(0)}(\eta)}{\sqrt{\Sigma(t)}}
\end{aligned}
$$

where $d \ln \Sigma(t) / d t=2 r(t)+\alpha(t, 0)(\Sigma(t))^{-1}$ is used.

Once we substitute (21), (22), (23), and (24) into (6), and equate the coefficients of $\epsilon^{n}$ on each side, the last terms on the RHS of (21) and (24) are cancelled out. and the PDE of $\varphi_{n}$ is finally obtained as follows.

Theorem 1 The PDE of $\varphi_{n}(n=1,2, \ldots)$ is given by

$$
\begin{aligned}
& \left(\partial_{t} \varphi_{n}\right)(t, \eta) \\
& =\frac{d \theta(t)}{d t} \mathcal{B}_{\eta}^{+} \mathcal{B}_{\eta}^{-} \varphi_{n}(t, \eta) \\
& +\sum_{m=0}^{n-1}\left(\begin{array}{c}
n \\
m
\end{array}\right) \frac{\left(\partial_{y}^{n-m} \alpha\right)(t, 0)}{2 \Sigma(t)}(\Sigma(t))^{(n-m) / 2} \\
& \quad \times\left(\mathcal{B}_{\eta}^{+}\right)^{2}\left(\mathcal{B}_{\eta}^{+}+\mathcal{B}_{\eta}^{-}\right)^{n-m} \varphi_{m}(t, \eta),
\end{aligned}
$$

where $\omega(t)=\sqrt{\Sigma(t)} e^{-\int_{0}^{t} r(u) d u}=e^{-\theta(t)}$. The initial condition of (25) is given by

$$
\lim _{t \downarrow 0} \int_{-\infty}^{\infty} h(\eta \sqrt{\Sigma(t)}) \varphi_{n}(t, \eta) \psi^{(0)}(\eta) d \eta=0 .
$$

It should be emphasized that the homogeneous term on the RHS of (25) is an operation by operator $\mathcal{B}_{\eta}^{+} \mathcal{B}_{\eta}^{-}$ whose eigenfunctions are Hermite polynomials. Furthermore, the nonhomogeneous terms on the RHS of (25) only include $\varphi_{m}$ for $m$ smaller than $n$. Therefore, we may be able to solve (25) recursively. This recursive structure is just a PDE-counter part to that found by $[1,2]$ as seen in Section 3. Moreover, owing to properties (19) and (20), if all of $\varphi_{n}$ are expanded by Hermite polynomials, so is the RHS of (25). Hence, we keep term $\left(\mathcal{B}_{\eta}^{+}+\mathcal{B}_{\eta}^{-}\right)^{n-m}$ on the RHS of (23) and (25) instead of replacing it with $\eta^{n-m}$. In the next section, we will see that the solution of (25) are expanded by the Hermite polynomials that are eigenfunctions of $\mathcal{B}_{\eta}^{+} \mathcal{B}_{\eta}^{-}$.

\section{Calculation of $\varphi_{n}$ with ODEs}

It is well-known that in the weighted real Hilbert space $L^{2}\left(\mathbb{R}_{\eta}, \psi^{(0)}\right)$ with inner product $\langle\psi, \varphi\rangle:=$ $\int_{-\infty}^{\infty} \psi(\eta) \varphi(\eta) \psi^{(0)}(\eta) d \eta$ for $\psi, \varphi \in L^{2}\left(\mathbb{R}_{\eta}, \psi^{(0)}\right)$, $\left\{H_{n}^{\infty}\right\}_{n=0}^{\infty}$ satisfy the orthogonality $\left\langle H_{m}, H_{n}\right\rangle=n ! \delta_{m, n}$, where $\delta_{m, n}$ is the Kronecker delta, and they form a complete orthogonal system of $L^{2}\left(\mathbb{R}_{\eta}, \psi^{(0)}\right)$.

Let us try to identify the solution of (25) expanded by Hermite polynomials with unknown functions $a_{n l}$ of $t$ in the form of

$$
\varphi_{n}(t, \eta)=\sum_{l=0}^{\infty} \frac{a_{n l}(t)}{(\omega(t))^{l}} H_{l}(\eta) .
$$

Then, we can clearly see that the initial condition of $a_{n l}$ for any $n, l$ is given by

$$
a_{n l}(0)=\lim _{t \downarrow 0} \frac{(\omega(t))^{l}}{l !}\left\langle\varphi_{n}(t, \cdot), H_{l}\right\rangle=0 .
$$

The first equality follows from the orthogonality of 
$\left\{H_{n}\right\}_{n=0}^{\infty}$ and the expansion (27). The last equality is the consequence of (26) by remembering that $H_{n}(\eta)$ can be written in the polynomial form of $\sum_{i=0}^{[n / 2]} c_{n, n-2 i} \eta^{n-2 i}$ where $c_{n, n-2 i}$ is the appropriate constant and $[x]$ denotes the greatest integer less than or equal to $x$.

We will now demonstrate how to determine $a_{n l}$ recursively by the ODEs for $n=1$ and 2. For $n=1$, (25) becomes

$$
\left(\partial_{t} \varphi_{1}\right)(t, \eta)=\frac{d \theta(t)}{d t} \mathcal{B}_{\eta}^{+} \mathcal{B}_{\eta}^{-} \varphi_{1}(t, \eta)+\frac{\left(\partial_{y} \alpha\right)(t, 0)}{2 \sqrt{\Sigma(t)}} H_{3}(\eta)
$$

where $\varphi_{0}(t, \eta)=H_{0}(\eta)=1$ and $\left(\mathcal{B}_{\eta}^{+}\right)^{2}\left(\mathcal{B}_{\eta}^{+}+\right.$ $\left.\mathcal{B}_{\eta}^{-}\right) \varphi_{0}(t, \eta)=H_{3}(\eta)$. By substituting $(27)$ with $n=1$ into the above PDE and taking the inner product with $H_{l}$ on both sides, we obtain the ODE for $a_{1 l}$ as

$$
\frac{d a_{13}(t)}{d t}=\frac{\left(\partial_{y} \alpha\right)(t, 0)}{2 \sqrt{\Sigma(t)}}(\omega(t))^{3}
$$

and $d a_{1 l}(t) / d t=0$ for $l \neq 3$ due to the orthogonality of $\left\{H_{n}\right\}_{n}$. These are directly solved under initial condition (28) and the results are $a_{13}(t)=$ $\int_{0}^{t}(1 / 2)\left(\partial_{y} \alpha\right)(u, 0) \Sigma(u) e^{-3 \int_{0}^{u} r(s) d s} d u$ and $a_{1 l}(t)=0$ for all $l \neq 3$. Hence, $\varphi_{1}(t, \eta)=(\omega(t))^{-3} a_{13}(t) H_{3}(\eta)$, which involves $H_{3}$ only as a function of $\eta$.

For $n=2$, the substitutions of $\varphi_{0}(t, \eta)=1$ and $\varphi_{1}$ obtained above into (25) lead to

$$
\begin{aligned}
\left(\partial_{t} \varphi_{2}\right)(t, \eta)=\frac{d \theta(t)}{d t} & \mathcal{B}_{\eta}^{+} \mathcal{B}_{\eta}^{-} \varphi_{2}(t, \eta) \\
& +\frac{\left(\partial_{y} \alpha\right)(t, 0)}{\sqrt{\Sigma(t)}(\omega(t))^{3}} a_{13}(t)\left(H_{6}(\eta)+3 H_{4}(\eta)\right) \\
& +\frac{\left(\partial_{y}^{2} \alpha\right)(t, 0)}{2}\left(H_{4}(\eta)+H_{2}(\eta)\right)
\end{aligned}
$$

This time, as the nonhomogeneous terms on the RHS of the PDE include $\mathrm{H}_{2}, \mathrm{H}_{4}$, and $\mathrm{H}_{6}$, we anticipate that $a_{2 l}$ will vanish except for $l=2,4$, and 6 . In fact, by using the same procedure as for $n=1$, one can show that

$$
\begin{aligned}
& \frac{d a_{22}(t)}{d t}=\frac{\left(\partial_{y}^{2} \alpha\right)(t, 0)}{2}(\omega(t))^{2} \\
& \frac{d a_{24}(t)}{d t}=3 \frac{\left(\partial_{y} \alpha\right)(t, 0) a_{13}(t)}{\sqrt{\Sigma(t)}} \omega(t)+\frac{\left(\partial_{y}^{2} \alpha\right)(t, 0)}{2}(\omega(t))^{4}, \\
& \frac{d a_{26}(t)}{d t}=\frac{\left(\partial_{y} \alpha\right)(t, 0) a_{13}(t)}{\sqrt{\Sigma(t)}}(\omega(t))^{3}
\end{aligned}
$$

and $d a_{2 l}(t) / d t=0$ for $l \neq 2,4,6$. Note that these ODEs depend on $a_{13}$ only due to the recursive nature of (25). Under initial condition (28), they are immediately solved, and $\varphi_{2}(t, \eta)$ involves $H_{2}(\eta), H_{4}(\eta)$, and $H_{6}(\eta)$ only. Summarizing all the results obtained above, we finally attain the expansion

$$
\begin{aligned}
& p^{(\epsilon)}(t, y) \\
& =\left[1+\epsilon \frac{a_{13}(t)}{(\omega(t))^{3}} H_{3}(\eta)+\frac{\epsilon^{2}}{2}\left(\frac{a_{22}(t)}{(\omega(t))^{2}} H_{2}(\eta)\right.\right. \\
& \left.\left.\quad+\frac{a_{24}(t)}{(\omega(t))^{4}} H_{4}(\eta)+\frac{a_{26}(t)}{(\omega(t))^{6}} H_{6}(\eta)\right)\right] p^{(0)}(t, y) \\
& \quad+o\left(\epsilon^{2}\right)
\end{aligned}
$$

where $\eta=y / \sqrt{\Sigma(t)}$. By a straightforward calculation, one can show that this expansion is identical to (4) that appears in Theorem 2.1 of [1] and in Theorem 3.2 of [2] at least up to second order of $\epsilon$.

In principle, we can calculate any higher order terms above $\epsilon^{2}$ by utilizing (25) recursively. Finally, next statement shows the order in which Hermite polynomials appear in $\varphi_{n}(t, \eta)$ so that $g_{n}$ can be expressed explicitly.

\section{Theorem 2 It holds that}

$$
g_{n}(t, y)=\left\{\begin{array}{cc}
\sum_{l \text { is even, and } l \geq 2}^{3 n} \frac{a_{n l}(t)}{(\omega(t))^{l}} H_{l}(\eta) & \text { if } n \text { is even, } \\
\sum_{l \text { is odd, and } l \geq 3}^{3 n} \frac{a_{n l}(t)}{(\omega(t))^{l}} H_{l}(\eta) & \text { if } n \text { is odd, }
\end{array}\right.
$$

where $\eta=y / \sqrt{\Sigma(t)}$.

Theorem 2 can be proved by mathematical induction in $n$. We have shown that it holds for $n=1,2$. Furthermore, we can show that if it holds for $n=2 i(i \geq 1)$, it also holds for $n=2 i+1$ (odd) and $n=2 i+2$ (even).

As is reviewed in Section 3, an expression with Hermite polynomials has already been obtained by [2]. However, in the expression there is a possibility of redundancy in the computation, some of which will eventually be zero since it has not ever been clarified which order of Hermite polynomials appear in the expression. Theorem 2 above shows which terms are not zero.

It should be noted that if the drift term of the underlying asset price process differs from a linear function $r(t) S_{t}^{(\epsilon)}$ as given in (1), the expression in Theorem 2 will be more complicated.

\section{Conclusion and remarks}

We have demonstrated how an eigenfunction expansion approach works to derive asymptotic expansions for the case of one risky asset by making full use of Hermite polynomials. In particular, Theorem 2 implies that our approach enables us to carry out a more efficient computation by focusing on an expansion of the PDF. In future, we will also study a case with multi-assets treated in [2].

\section{Disclaimer}

All opinions expressed by the authors of this letter are solely the authors' opinions and do not reflect the opinions of Mizuho Securities Co. Ltd.

\section{References}

[1] A. Takahashi, An asymptotic expansion approach to pricing financial contingent claims, Asia-Pac. Financ. Mark., 6 (1999), $115-151$.

[2] A. Takahashi, K. Takehara, and M. Toda, A general computation scheme for a high-order asymptotic expansion method, Int. J. Theor. Appl. Finance, 15 (2012), 1250044.

[3] A. Takahashi and T. Yamada, A remark on approximation of the solutions to partial differential equations in finance, in: Recent Advances in Financial Engineering 2011, pp 133-181, World Scientific, Singapore, 2012.

[4] A. Arai, Inequivalent Representations of Canonical Commutation and Anti-Commutation Relations, Springer, Singapore, 2020 . 